\title{
Oxidative Stress: A Double Edged Sword
}

\author{
Kausar Abbas $^{1}$, Ufaque Batool ${ }^{1 *}$, Abdul Shakoor Memon ${ }^{2}$, and Sana Soomro ${ }^{1}$ \\ ${ }^{1}$ Department of Physiology, BMSI, Jinnah Post Graduate Medical Centre, Karachi, Pakistan \\ ${ }^{2}$ Department of Physiology, Sir Sayed College of Medical Sciences, Karachi, Pakistan
}

Received: June 202021

Accepted: June 29, 2021

\begin{abstract}
Oxidative stress (OS) in simple words is defined as a state of imbalance between antioxidant and pro-oxidants defenses. The purpose of this article is to find out the connection of oxidative stress and free radical species with different aspects of human health. Owing to its harmful effects on proteins and nucleic acids, oxidative stress causes chronic diseases such as cardiovascular and neurodegenerative diseases, diabetes and cancer. It highlights the impacts of antioxidants and pro-oxidants particularly on fertility and infertility. It also focuses on the adverse effects caused by the long-term exposure to pro-oxidant factors leading to structural defects of mitochondrial DNA. As modern life style consists of more reliance on the processed foods and lack of physical activity, a nutrition deficiency, which is common in the present lifestyle, is also one of the reasons for oxidative stress to cause inflammation. However, this review also focuses on how diet affects and triggers inflammation. Redox mechanism with potential threats to health of mankind is discussed, how mere stress can provoke biological stress responses leading to development of disease or metabolic errors. Methods for reduction of oxidative stress are discussed in this review. It also highlights mitochondria as an aging factor as many ROS, particularly mitochondria ROS contribute directly to aging in human body. We will be discussing the recent findings in the oxidative stress field and its negative and positive impacts on human health.
\end{abstract}

Keywords: Oxidative Stress (OS), Reactive oxygen species (ROS), Reactive nitrogen species (RNS), Free radicals (FR), Redox (reduction-oxidation), Human health, antioxidants, pro oxidants

*Correspondence: Ufaque Batool, Department of Physiology, BMSI, Jinnah Post Graduate Medical Centre, Karachi, Pakistan. Tel: 03313436112 Email: ufaque.batool@gmail.com

\section{INTRODUCTION}

In the present era of technology and advancement, where our physical activities are replaced by machines, organic diet with the processed foods and environment is polluted with the burning of fuel. Factors like exposure to pollution, eating inorganic diet, and avoiding exercise give rise to several diseases and health issues

In order to improve our health, it is vital for mankind to fight against these factors. At present, without any doubt, we are witnessing an upsurge in health related problems like cardiovascular diseases, hypertension, diabetes, autoimmune disease and different kind of cancers and maladies. These all diseases are related to the factors mentioned above.

Latest medical surveys and researches have proven that oxidative stress serves as a potential tool for the control of these chronic maladies [1].

\section{Oxidative Stress:}

Oxidative stress is characterized by the imbalance between production and degradation of reactive oxygen species or reactive nitrogen species. ROS is metabolic byproduct of biological system [2].The production of ROS in human body, although in a small quantity, is very crucial as many processes like protein phosphorylation; activation of transcriptional factors like immunity and so on, depends on it.

ROS are mainly produced by mitochondria as a normal functioning of the body, but the antioxidant-present in the body acts in defense by preventing the excessive formation of ROS that could otherwise damage the body. Antioxidants form ROS, when body needs them and these ROS are also destroyed by them, when they create threat to human health. 
The concept of oxidative stress was first used by researchers in medicine in 1985. But unfortunately not many studies were done after that time. Presently, many researches are being carried out on the said topic around the globe, making people more aware of benefits and hazards of these antioxidants and oxidants [4,5]. In a nutshell, the oxidative stress and excessive accumulation of ROS radicals cause serious health hazards.

\section{Oxidative Stress and Homeostasis}

Each cell of human body has to maintain a condition of homeostasis between the oxidants and antioxidants species [6]. About 3\% of pulmonary oxygen is converted into ROS [1]. Only excessive formation of ROS and other free radicals are problematic, otherwise, their routine production are very essential as they help in the formation of ATP and contribute to the other normal functioning of human body. Its excess cause disturbance in normal phenomenon [7]. These free radicals are potential risk to mankind. ROS is simultaneously produced from generation of ATPs, although our body needs ATPs to carry out normal physiological functions. In oxidative stress, mitochondria form more ATPs to subside the effects of Oxidation from body, but more ATPs mean more ROS are formed. These ROS will circulate in cell, causing activation of reactive nitrogen compounds. Presence of nitrogen reactive compounds combining ROS cause self-damage of tissue by implementing genes in DNA and mitochondrial enzymes. Over production ROS will work as stimuli for Mitochondrial Toxicity.

\section{Physiological role of Stress:}

Oxidative stress has a dual role. Physiologically it tells how our body response toward oxidation and evolutionary it show how mutation in genes occurred from persistent tissue damage caused by oxidative stress. How evolution of body at DNA level is carried out by ROS, which is a vital companion of all normal processes. [11]

Our body need free radicals and reactive metabolites to carried out physiological functions, so it would not be beneficial to completely suppress free radical production [12].

Despite harmful effects of free radical, it has some beneficial role as well. Free radical work as a microbicidal system, its unbeatable role as a phagocyte is highly needed in wear and tear system of body. At present studies are carried out in aspect of their transduction role in signal regulation in intercellular information [14].

Today we are undergoing a situation where chronic health ailment like cardiovascular diseases and many others are becoming common affecting the morality rate worldwide [14].Central nervous system become a easy victim of free radical damage because it has small antioxidant capacity to resist the damage [19].

\section{Oxidative stress and altered immune functions:}

It is a well-established fact that the immune system and oxidative stress work together. Without oxidative stress, our immunity is incomplete. The immune system uses oxidants along with ROS and RNS to kill pathogens. An enzyme, called the nicotinamide adenine dinucleotide phosphate $\left(\mathrm{NADP}^{+}\right)$, produces ROS when immunity is compromise [20,21]. The production of ROS in excess quantity creates more oxidation within the body, leading to overcrowding of Free radicals. Presence of free radicals trigger process of autolysis of white blood cell, thus compromising body defense, and ending in autoimmune diseases.

The oxidative modification of the proteins altar the antigen profile and increase antigenicity [22]. There are many examples of auto immune diseases that are caused by oxidative modification of self-proteins these includes; systemic lupus erythematoses (SLE), diabetes mellitus and diffuse scleroderma among others [23, 24].Besides, it poses serious threats to some tissues. One common example is insulin producing beta cells in the islets of Langerhans 
[26]. Psychological stress plays a fundamental role in development of autoimmune pathogenesis along other stress hormones[27].

\section{Oxidative stress and cancer:}

Carcinogenesis is the progressive detritions, initiated by altered biological process of host system. It can cause permanent damage to the susceptible host, if not treated beforehand. The initial steps involved in development of cancer, mutation or aging are outcomes of oxidative damages happening to cell DNA. The oxidized by products, which can leads to altered gene variation, mutation or can leads to cancer. DNA strands damage is caused by alteration in purine and pyrimidine bonding, which is initiated by ROS. In regular cellular mechanism Redox metals and ROS control DNA Repair mechanism by co habiting with enzymes like catalyse, peroxidases and superoxide dismutase. The leftover of free radicals are carried out by vitamin $\mathrm{E}, \mathrm{C}$ and glutathione along with these enzymes. The oxidative stress disables this apoptosis pathway of Redox, with no ability to repair DNA, and over production of ROS within body cause development of carcinogenesis [28]. Oxidative stress produced factors like TNF, proteases, ROS, and kinases, are progenitors of tumor growth and proliferation. These factors cause cancer to spread in lymphatics by membrane degradation, and invading to neighbouring tissues by disturbing vasculature of cells [29,31]. In present times, incidence of thyroid cancer is increasing rapidly, the reason behind is increased free radical generation, as more and more people are exposed to radiations [32]. Many studies have proven oxidative stress as the causative agent for development of cancer [41]. Incidence of cancers like colorectal, breast, pancreatic cancers have thrice more tendency to develop with diabetes type 1 and obesity. This relation implements the role on leptin [42], insulin/ insulin- like growth factor-1, adiponectin as a coherent to inflammation with cancer and obesity. More ROS production is also linked with fat accumulation and oxidative stress. Its elevation cause wide range of transcription factors such as $\mathrm{Wnt} / \beta$-catenin, NF- $\mathrm{KB}$, and nuclear factor E2-related factor 2 (Nrf2) to malfunction resulting in activation of many inflammatory pathways.

\section{Obesity and Adipocyte Dysfunction:}

Obesity is chronic inflammatory disease, which is modulated by oxidative stress caused within the body by infiltration of adipose tissue, oversupply of carbohydrate and fats. Inflammatory cytokines involved in fat metabolism are close associated with plethora of abdominal obesity. Oxidative stress plays a vital role in inflammation caused by obesity. Excessive intake of carbohydrates, protein, lipids and nutrients, metabolic disturbances or monocytes infiltrates including, t-cells, mast cells, and macrophages are modulated by adipocytes, and cause disturbance of adipose functions [33, 34]. A study by Lim [35] stated that these nutrients activate protease receptor 2 expression. Study also concluded that hypertrphic adipocyes yield from HFD cause production of ROS.

\section{Diabetes and OS:}

Diabetes type 2 currently represents nearly $90-95 \%$ of Diabetes Mellitus cases. It is the most common, complex and progressively accomplished by many complications if not controlled. Among many other neuropathy, retinopathy, nephropathy, micro and macro vascular damages are commonest.

Diabetes mellitus is caused by multifactoral factors, among others oxidative stress is classified as major risk factors in its development. Oxidative environment modify insulin insensitivity, either by elevating insulin resistance or by altering glucose tolerance. In both condition body is exposed to hyperglycemic state, which leads to progression of further oxidative state development [36]. Complications of Diabetes mellitus are also associated with OS [37]. 


\section{Cardiovascular disease and OS}

Cardio vascular system is also implicated by oxidative stress. Its development and functions are highly influenced by over production of ROS and reduced presence of antioxidants [38]. Imbalance of this equilibrium of antioxidants and ROS is caused by HFD. Oxidative state of heart leads to development of many complications. OS has potential to cause mitochondrial dysfunction, more ROS production will produce more fatty acid oxidation and increase activity of NADPH, disorders like endothelial dysfunctions, less bioavailability of vasodilators, and endothelial derived contractile factors promotes development of atherosclerotic disease [39, 40]. Ilkun and Boudinna have described the complex mode of actions in cardiac pathology. They stated that dysfunction of autophagy, lipid accumulation cause stiffness and fibrosis of arteries. Further persistent of oxidative state will lead to damage at mitochondrial level.

\section{Rheumatoid Arthritis and OS}

Many studies have been carried out in respect of RA association with LPO, protein oxidation and oxidative DNA damage, in more than $85 \%$ cases with RA, these damages and oxidation are found to be increased with more severity of ROS presence. As we all know inflammation is first patho-physiological response of RA mechanism. Macrophages and neutrophils make immunity by producing ROS like $02-$ and $\mathrm{H} 2 \mathrm{O} 2$ which will cause inflammation s first line defense, but when mitochondrial damage cause over production ROS, it will be free radical injury to the cell. Mitochondrial ROS produce pro inflammatory cytokines IL-B, Il-6 and TNF-a. It also participates in inflammation agonist signaling. This signaling pathways will too leads to production of ROS, because ROS produced by NADPH oxidase enzyme pathways are regulated by polymorphonuclear neurtrophils, which are pro creators of OS through inflammation.

\section{Neuro degenerative Disease and OS}

Neurodegenerative Diseases like parkinson's [45], Alzheimer's [46], multiple sclerosis and depression are highly interconnected with OS with ageing being the main link between these two entities. The neural tissue damage is mainly result from mitochondrial dysfunction. OS is known as a trigger for development of mitochondrial damage and produce neuro toxins end product which further worsen the neural health of human being. B-amyloid a neuro peptide toxin which is produced by ROS, play a great role in alzheimer's disease development and progression [47].

\section{Respiratory Disease and OS:}

Oxidative stress can cause local and systemic inflammation, from conclusion of many studies, researcher have narrated that lung problems like asthma and chronic obstructive pulmonary diseases are caused by inflammation from oxidants, these oxidants enhance inflammation by activating pathways of kinease and transcription factors like NF-kappa B and AP-1.

\section{Kidney Diseases and OS:}

Oxidation stress is pro creator of many kidney diseases, problems like glomerulo- and tubuleinterstitial nephritis, renal failure, proteinuria, uremia, disorders of renal apparatus and many like them, they all are hugely affected by Oxidative stress because of production of ROS. The mode of action is same, it trigger inflammatory cytokine production that leads to initial inflammation to happen. TNF-alpha and IL-1b as a pro inflammatory mediators play predominant role in initiation of inflammation and, transcription factor NF- $\kappa \mathrm{B}$ is used to 
sustain the process of inflammation. In end state reaction, TGF- beta production works on extracellular matrix synthesis so that when kidney tissue receive stimuli from oxidation, it starts initial inflammation. This inflammation deepen with persistent formation of excessive fibrotic tissue results from impair organ functions and ultimate cause renal failure

\section{Sexual Maturation and OS:}

Oxidative stress have very potential control on our hormones, especially reproductive hormones are grossly affected by Stress. One of the Stress hormones, cortisol is increased in body, if body remains in oxidative phase for so long. Many studies suggested that oxidative stress can delay sexual maturation. This seems to be true, when $\mathrm{Cd}$ is exposed in young forms more free radicals and further enhances oxidative stress. The oxidative stress when expose in women can leads to infertility, persistent presence of ROS and OS in body lead to hormonal imbalance, especially FSH and LH are heighted affected by OS, and these hormones are very necessary for normal process of reproduction, as FSH is needed for maturation of Oocytes and LH works on uterine endometrium, imbalance of LH cause thickening of uterine walls, making it impossible for implantation of egg [50]. Beside these effects in females, oxidative stress affects quality of sperms.

\section{CONCLUSION:}

Oxidative stress and free radicals are very deleterious to mankind health. They play potential role in development, progression, and onset of many pathologies and diseases, alternating normal physiological mechanism, going from the simple to the complex level of diseases, form simple immunity change to pathological involvement of cancer. Recently antioxidants became attention of many researchers, as this group of compound play great role in counteracting oxidative stress reactions thus preventing diseases and promoting health of mankind. From results of many studies, we can conclude that oxidative stress and free radical are hazardous to human health. Anti oxidants and pro oxidants can be used as a treatment tool. The oxidative stress can be reduced in 3 ways, firstly by decreasing the exposure of environmental pollutants, secondly by increasing the antioxidants both endogenously and exogenously, and lastly by strengthen the mechanism of mitochondrial oxidative generation production and efficiency. This article also provide evidence of how antioxidants can delay insulin resistance onset, how it can prevent tissue injury, and delay cancer proliferation.

\section{FUTURE PROJECTIONS:}

Oxidative stress is closely associated with metabolic ailments, fats consumption, high proteins diets, food with high carbs contents can trigger this stress. This article clearly identify how oxidative stress is a linkage between mediating pathways of obesity and it's related errors. Only loose loop in this field is very little work in done in prevention aspects of these metabolic errors. Antioxidants play vital role in this regards yet not very consistent researches and studies has been done in this field to support this action of antioxidants in prevention and delaying the process of disease or metabolic errors. Although animal studies have been done worldwide on redox mechanism, no such work is done Pakistan so far, even on animals.

More work on randomized clinical triads for redox phenomenon at cellular levels should be done to further evaluate long term effects oxidative stress on human health, molecular bioenergetics will further propel concept of oxidative stress. Following quote from Hans Selye best describes concept of Oxidative stress; "we can enormously stretch our life length, if only we could see and measure Stress". 


$\begin{array}{ll}\text { Abbreviations: } & \\ \text { Oxidative Stress } & \text { (OS), } \\ \text { Reactive oxygen Species } & \text { (ROS), } \\ \text { Reactive nitrogen species } & \text { (RNS), } \\ \text { Free radicals } & \text { (FR), } \\ \text { nicotinamide adenine dinucleotide phosphate } & \text { (NADP), } \\ \text { reduction-oxidation } & \text { (Redox), } \\ \text { systemic lupus erythematoses } & \text { (SLE), } \\ \text { deoxyribonucleic acid } & \text { (DNA), } \\ \text { high fat diet } & \text { (HFD), } \\ \text { cardiovascular system } & \text { (CVS), } \\ \text { Rheumatoid Arthritis } & \text { (RA), } \\ \text { lipid peroxidation } & \text { (LPO), } \\ \text { Cadmium } & \text { (Cd) }\end{array}$

ETHICS APPROVAL AND CONSENT TO PARTICIPATE

Not applicable.

HUMAN AND ANIMAL RIGHTS

Not applicable.

CONSENT FOR PUBLICATION

Not applicable.

AVAILABILITY OF DATA AND MATERIALS

None.

FUNDING

None.

\section{CONFLICT OF INTEREST}

The authors declare no conflict of interest, financial or otherwise.

\section{ACKNOWLEDGEMENTS}

I am very thankful to Dr kausar Abbas Saldera, for her guidance and advice for this article.

\section{References:}

1. Woodruff, T. J., Janssen, S. J., GuilletteJr, L. J., \&Giudice, L. C. (Eds.). (2010). Environmental impacts on reproductive health and fertility. Cambridge University Press.

2. Ahmed, H. M., Khan, M., Yasmin, F., Jawaid, H., Khalid, H., Shigri, A., ... \&Hasan, C. A. (2020). Awareness regarding causes of infertility among out-patients at a tertiary care hospital in Karachi, Pakistan. Cureus, 12(4)2020.

3. H. Sato, H. Shibata, T. Shimizu, S. Shibata, H. Toriumi, and T. Ebine, "Differential cellular localization of antioxidant enzymes in the trigeminal ganglion," Neuroscience, vol. 248, pp. 345-358, 2013.

4. J. Navarro-Yepes, L. Zavala-Flores, A. Anandhan, F. Wang, M. Skotak, and N. Chandra, "Antioxidant gene therapy against neuronal cell death," Pharmacology \& Therapeutics, vol. 142, pp. 206-230, 2014

5. Alam, F., Khan, T. A., Ali, R., Tariq, F., \& Rehman, R. (2020). SIRTI and cortisol in unexplained infertile females; a cross sectional study, in Karachi Pakistan. Taiwanese Journal of Obstetrics and Gynecology, 59(2), 189-194.

6. G. Poli, G. Leonarduzzi, F. Biasi, and E. Chiarpotto, "Oxidative stress and cell signalling," Current Medicinal Chemistry, vol. 11, no. 9, pp. 1163-1182, 2004 
7. Hussain, R., Khaliq, S., Raza, S. M., Khaliq, S., \& Lone, K. P. (2018). Association of TP53 codon 72 polymorphism in women suffering from endometriosis from Lahore, Pakistan. J Pak Med Assoc, 68, 224-230.

8. B. Meier, H. H. Radeke, S. Selle et al., "Human fibroblasts release reactive oxygen species in response to interleukin-1 or tumour necrosis factor- $\alpha$," Biochemical Journal, vol. 263, no. 2, pp. $539-545,1989$

9. J. P. Fulgencio, C. Kohl, J. Girard, and J. P. Pegorier, "E' ffect of metformin on fatty acid and glucose metabolism in freshly isolated hepatocytes and on specific gene expression in cultured hepatocytes," Biochemical Pharmacology, vol. 62, no. 4, pp. 439-446, 2001.

10. M. Birringer, "Hormetics: dietary triggers of an adaptive stress response," Pharmaceutical Research, vol. 28, no. 11, pp. 2680-2694, 2011

11. B. Halliwell and J. M. C. Gutteridge, Free Radicals in Biology and Medicine, Oxford University Press, Oxford, UK, 3rd edition, 2007.

12. Z. Dura 'ckov `a, L'. Bergendi, and J. ' $\mathrm{C}^{`}$ arsky, Eds., ' Free Radicals and Antioxidants in Medicine (II), SAP, Bratislava, Slovakia, 1999.

13. A. H. Klimp, E. G. E. De Vries, G. L. Scherphof, and T. Daemen, "A potential role of macrophage activation in the treatment of cancer," Critical Reviews in Oncology and Hematology, vol. 44, no. 2, pp. 143-161, 2002.

14. Z. Dura ckov`a, "Some current insights into oxidative stress," ' Physiological Research, vol. 59, no. 4, pp. 459-469, 2010

15. J. Racek, V. Treska, V. Krizan, V. Holecek, and Z. Jerabek, "The significance of free radicals in operations of acute ischaemia of the limbs," Zeitschrift fur klinischeChemie und klinische " Biochemie, vol. 3, pp. 103-105, 1995

16. A. Cherubini, C. Ruggiero, M. C. Polidori, and P. Mecocci, "Potential markers of oxidative stress in stroke," Free Radical Biology and Medicine, vol. 39, no. 7, pp. 841-852, 2005.

17. K. P. Singh, A. H. Ahmad, S. K. Hore, V. Singh, M. Lohani, and A. Rahal, "Effect of Emblicaofficinalis on antioxidative and haematological parameters following mercury induced toxicity," in Proceedings of the 6th Annual conference of ISVPT, Patna, India, November 2006.

18. N. Valero, J. Mosquera, G. Anez, A. Levy, R. Marcucci, and D. M. Melchor, "Differential oxidative stress Induced by dengue virus in monocytes from human neonates, adult and elderly individuals," PLoS ONE, vol. 8, no. 9, Article ID e73221, 2013

19. S. S. Leonard, G. K. Harris, and X. Shi, "Metal-induced oxidative stress and signal transduction," Free Radical Biology and Medicine, vol. 37, no. 12, pp. 1921-1942, 2004

20. M. B. Hampton, A. J. Kettle, and C. C. Winterbourn, "Inside the neutrophil phagosome: oxidants, myeloperoxidase, and bacterial killing," Blood, vol. 92, no. 9, pp. 3007-3017, 1998.

21. B. M. Babior, "NADPH oxidase: an update," Blood, vol. 93, no. 5, pp. 1464-1476, 1999

22. M. E. Allison and D. T. Fearon, "Enhanced immunogenicity of aldehyde bearing antigens: a possible link between innate and adaptive immunity," European Journal of Immunology, vol. 10, pp. 2881-2887, 2000.

23. L. Casciola-Rosen, F. Wigley, and A. Rosen, "Scleroderma autoantigens are uniquely fragmented by metal-catalyzed oxidation reactions: implications for pathogenesis," Journal of Experimental Medicine, vol. 185, no. 1, pp. 71-79, 1997.

24. B. Buttari, E. Profumo, V. Mattei et al., "Oxidized $\square 2$ - glycoprotein I induces human dendritic cell maturation and promotes a T helper type 1 response," Blood, vol. 106, no. 12, pp. 3880-3887, 2005.

25. S. M. Trigwell, P. M. Radford, S. R. Page et al., "Islet glutamic acid decarboxylase modified by reactive oxygen species is recognized by antibodies from patients with type 1 diabetes mellitus," Clinical and Experimental Immunology, vol. 126, no. 2, pp. 242-249, 2001.

26. S. E. Gandy, M. G. Buse, and R. K. Crouch, "Protective role of superoxide dismutase against diabetogenic drugs," Journal of Clinical Investigation, vol. 70, no. 3, pp. 650-658, 1982

27. L. Stojanovich, "Stress and autoimmunity," Autoimmunity Reviews, vol. 9, no. 5, pp. A271A276, 2010. 
28. T. B. Kryston, A. B. Georgiev, P. Pissis, and A. G. Georgakilas, "Role of oxidative stress and DNA damage in human carcinogenesis," Mutation Research, vol. 711, no. 1-2, pp. 193$201,2011$.

29. A. R. Davalos, J. Coppe, J. Campisi, and P. Desprez, "Senescent cells as a source of inflammatory factors for tumor progression," Cancer and Metastasis Reviews, vol. 29, no. 2 , pp. 273-283, 2010.

30. F. Innocenti, N. J. Cox, and M. E. Dolan, "The use of genomic information to optimize cancer chemotherapy," Seminars in Oncology, vol. 38, no. 2, pp. 186-195, 2011.

31. B. Vire, A. David, and A. Wiestner, "TOSO, the Fc $\square$ receptor, is highly expressed on chronic lymphocytic leukemia B cells, internalizes upon IgM binding, shuttles to the lysosome, and is downregulated in response to TLR activation," Journal of Immunology, vol. 187, no. 8, pp. 4040-4050, 2011.

32. G. Pellegriti, F. Frasca, C. Regalbuto, S. Squatrito, and R. Vigneri, "Worldwide increasing incidence of thyroid cancer: update on epidemiology and risk factors," Journal of Cancer Epidemiology, vol. 2013, Article ID 965212, 10 pages, 2013

33. T. M. Hagen, "Oxidative stress, redox imbalance, and the aging process,"Antioxidants and Redox Signaling, vol. 5, no. 5, pp. 503- 506, 2003.

34. N. Babbar and E. W. Gerner, "Targeting polyamines and inflammation for cancer prevention," Recent Results in Cancer Research, vol. 188, pp. 49-64, 2011.

35. D. S. Shames, J. D. Minna, and A. F. Gazdar, "DNA methylation in health, disease, and cancer," Current Molecular Medicine, vol. 7, no. 1, pp. 85-102, 2007

36. A. Ceriello, "Possible role of oxidative stress in the pathogenesis of hypertension," Diabetes Care, vol. 31, Supplement 2, pp. S181-S184, 2008.

37. Chen, X.-F., Wang, L., Wu, Y.-Z., Song, S.-Y., Min, H.-Y., Yang, Y., et al. (2018). Effect of puerarin in promoting fatty acid oxidation by increasing mitochondrial oxidative capacity and biogenesis in skeletal muscle in diabetic rats. Nutr. Diabetes 8, 1-13.

38. H. Bugger and E. D. Abel, "Molecular mechanisms for myocardial mitochondrial dysfunction in the metabolic syndrome," Clinical Science, vol. 114, no. 3, pp. 195-210, 2008

39. R. Lee, M. Margaritis, M. K. Channon, and C. Antoniades, "Evaluating oxidative stress in human cardiovascular disease: methodological aspects and considerations," Current Medicinal Chemistry, vol. 19, no. 16, pp. 2504-2520, 2012.

40. O. Ilkun and S. Boudina, "Cardiac dysfunction and oxidative stress in the metabolic syndrome: an update on antioxidant therapies," Current Pharmaceutical Design, vol. 19, no. 27, pp. 4806-4817, 2013

41. B. L. Tan, M. E. Norhaizan, K. Huynh, S. K. Yeap, H. Hazilawati, and K. Roselina, "Brewers' rice modulates oxidative stress in azoxymethane-mediated colon carcinogenesis in rats," World Journal of Gastroenterology, vol. 21, no. 29, pp. 8826-8835, 2015.

42. M. J. Khandekar, P. Cohen, and B. M. Spiegelman, "Molecular mechanisms of cancer development in obesity," Nature Reviews Cancer, vol. 11, no. 12, pp. 886-895, 2011.

43. T. Liu, L. Zhang, D. Joo, and S. C. Sun, "NF-kB signaling in inflammation," Signal Transduction and Targeted Therapy, vol. 2, no. 1, 2017.

44. A. C. Bulua, A. Simon, R. Maddipati et al., "Mitochondrial reactive oxygen species promote production of proinflammatory cytokines and are elevated in TNFR1-associated periodic syndrome (TRAPS)," The Journal of Experimental Medicine, vol. 208, no. 3, pp. 519-533, 2011.

45. O. Hwang, "Role of oxidative stress in Parkinson's disease," Experimental neurobiology, vol. 22, no. 1, pp. 11-17, 2013.

46. E. Tönnies and E. Trushina, "Oxidative stress, synaptic dysfunction, and Alzheimer's disease," Journal of Alzheimer's Disease, vol. 57, no. 4, pp. 1105-1121, 2017

47. D. A. Butterfield, "Amyloid $\beta$-peptide (1-42)-induced Oxidative Stress and Neurotoxicity: Implications for Neurodegeneration in Alzheimer's Disease Brain. A Review," Free Radical Research, vol. 36, no. 12, pp. 1307-1313, 2009

48. W. MacNee, "Oxidative stress and lung inflammation in airways disease," European Journal of Pharmacology, vol. 429, pp. 195-207, 2001. 


\section{Biosight 2021; 02(01): 4-12}

49. M. Interdonato, G. Pizzino, A. Bitto et al., "Cadmium delays puberty onset and testis growth in adolescents," Clinical Endocrinology, vol. 83, no. 3, pp. 357-362, 2015

50. Roychoudhury, S., Agarwal, A., Virk, G., and Cho, C. L. (2017). Potential role of green tea catechins in the management of oxidative stress-associated infertility. Reprod. Biomed. Online 34, 487-498. doi: 10.1016/j.rbmo.2017.02.006 